\title{
Genetic analysis and fine mapping of an enclosed panicle mutant esp2 in rice (Oryza sativa $\mathrm{L}$.
}

\author{
GUAN HuaZhong ${ }^{1,2^{*}}$, DUAN YuanLin ${ }^{1,2^{*}}$, LIU HuaQing ${ }^{3 *}$, CHEN ZhiWei $^{1,2}$, ZHUO Ming $^{1}$, \\ ZHUANG LiJun $^{1}$, QI WenMing ${ }^{1}$, PAN RunSen ${ }^{1,2}$, MAO DaMei ${ }^{1,2}$, ZHOU YuanChang ${ }^{1,2}$, \\ $\mathrm{WANG}$ Feng $^{3 \dagger}$ \& WU WeiRen ${ }^{1,2 \dagger}$ \\ ${ }^{1}$ Key Laboratory of Ministry of Education for Genetics, Breeding and Multiple Utilization of Crops, Fujian Agricultural \& Forestry University, \\ Fuzhou 350002, China; \\ ${ }^{2}$ Fujian Provincial Key Laboratory of Marker-Assisted Breeding of Rice, Fujian Agriculture and Forestry University, Fuzhou 350002, China; \\ ${ }^{3}$ Fujian Agricultural Science Research Institute, Fuzhou 350002, China
}

Received October 26, 2010; accepted February 23, 2011

\begin{abstract}
The phenomenon of panicle enclosure in rice is mainly caused by the shortening of uppermost internode. Elucidating the molecular mechanism of panicle enclosure will be helpful for solving the problem of panicle enclosure in male sterile lines and creating new germplasms in rice. We acquired a monogenic recessive enclosed panicle mutant, named as esp2 (enclosed shorter panicle 2), from the tissue culture progeny of indica rice cultivar Minghui-86. In the mutant, panicles were entirely enclosed by flag leaf sheaths and the uppermost internode was almost completely degenerated, but the other internodes did not have obvious changes in length. Genetic analysis indicated that the mutant phenotype was controlled by a recessive gene, which could be steadily inherited and was not affected by genetic background. Apparently, ESP2 is a key gene for the development of uppermost internode in rice. Using an $\mathrm{F}_{2}$ population of a cross between esp2 and a japonica rice cultivar Xiushui-13 as well as SSR and InDel markers, we fine mapped ESP2 to a 14-kb region on the end of the short arm of chromosome 1. According to the rice genome sequence annotation, only one intact gene exists in this region, namely, a putative phosphatidylserine synthase gene. Sequencing analysis on the mutant and the wild type indicated that this gene was inserted by a 5287-bp retrotransposon sequence. Hence, we took this gene as a candidate of ESP2. The results of this study will facilitate the cloning and functional analysis of ESP2 gene.
\end{abstract}

rice, enclosed panicle mutant, ESP2 gene, fine mapping

Citation: Guan H Z, Duan Y L, Liu H Q, et al. Genetic analysis and fine mapping of an enclosed panicle mutant esp2 in rice (Oryza sativa L.). Chinese Sci Bull, 2011, 56: 1476-1480, doi: 10.1007/s11434-011-4552-9

At present, the planting area of hybrid rice in China is up to $17330000 \mathrm{hm}^{2}$, accounting for more than half of the total planting area of rice, for which an area of $\sim 150000 \mathrm{hm}^{2}$ is needed to produce hybrid rice seeds each year [1,2]. The currently used indica rice male sterile lines all suffer the problem of panicle enclosure to some extent, with $30 \%-60 \%$ of the panicle enclosed by flag leaf sheath due to the length reduction of uppermost internode. To overcome

*These authors contributed equally to this work.

†Corresponding authors (email: wuwr@fjau.edu.cn; wf@fjage.org) the panicle enclosure so as to increase the yield of hybrid seeds, a common practice is to spray gibberellin $\left(\mathrm{GA}_{3}\right)$ at heading stage. However, this approach has the shortcomings of increasing cost, reducing seed quality and polluting environment, etc. [3]. Hence, genetically eliminating panicle enclosure in male sterile lines has long been an important goal of hybrid rice breeding.

Rutger et al. [4] identified a recessive mutant with over-elongated uppermost internode in the $F_{3}$ generation of a cross between two japonica rice cultivars and named the mutant as elongated uppermost internode (eui). Yang et al. 
[5] obtained 2 eui mutant genes by irradiation mutating the indica rice cultivar Xieqingzao-B; they named the 2 genes as euil and eui2, among which euil is allelic to the one firstly identified by Rutger et al. [4]. He et al. [6] found that the euil mutant contained high levels of GA and was more sensitive to exogenous GA. Zhu [7] and Zhu et al. [8] have cloned the genes EUI2 and EUI1, respectively, revealing that EUII is a member of the cytochrome P450 family, while EUI2 encodes an epoxide hydrolase, both of which function to reduce the synthesis of endogenous GA in rice. Loss of any one of them could result in the accumulation of GA and therefore makes the uppermost internode elongated. The characteristic of over-elongation of uppermost internode in eui mutants is potentially useful for overcoming the panicle enclosure of sterile lines and improving the pollination ability of restorer lines. However, practice has indicated that both euil and eui2 genes can not completely alleviate the panicle enclosure of rice sterile lines, implying that the level of endogenous GA synthesis in the uppermost internode of rice sterile lines is very low [5,9-12].

Therefore, promoting GA synthesis in uppermost internode might be a key to solve the problem of panicle enclosure in rice sterile lines. For this purpose, it is necessary to research the causes of panicle enclosure in rice. Five enclosed panicle mutants have been reported in rice [13-16], all of which showed the characteristics of reduced plant height, shortened uppermost internode and panicle enclosure. However, these mutants have not been deeply studied so far. Most of the studies remained at the level of morphological, cytological and classical genetic analyses. Only one mutant gene (shp6) has been mapped so far [13].

We obtained a monogenic recessive mutant esp 2 from the progeny of tissue culture in rice, which exhibited complete panicle enclosure. In this study, we performed morphological observation and genetic analysis on the mutant and conducted fine mapping of ESP2 gene, aiming to facilitate the cloning and functional analysis of the gene.

\section{Materials and methods}

\subsection{Plant materials}

The esp 2 mutant was found from the tissue culture progeny of indica rice cultivar Minghui-86. Other experimental materials included the wild-type Minghui-86, indica rice cultivar Zao-R974 and japonica rice cultivar Xiushui-13.

\subsection{Genetic analysis}

The esp2 mutant was crossed with Minghui-86, Zao-R974 and Xiushui-13, respectively. The segregation of normal plants and mutant plants in the $F_{2}$ populations of the three crosses was investigated at the heading stage, and the segregation ratios were tested using the method of chi-square test.

\subsection{Molecular markers}

The RM-series SSR markers available on the website Gramene (http://www.gramene.org/) were used for preliminary mapping of the target gene. In the region covering the target gene, new SSRs were identified by scanning the genome sequence of japonica cultivar Nipponbare (http://rapdb.dna. affrc.go.jp/download/) using the program SSRIT provided by Gramene, and InDels were identified by aligning the genome sequences of Nipponbare and indica cultivar 93-11 (http://rice.genomics.org.cn/rice/link/download.jsp) using the program BLAST. Specific primer pairs were designed to develop new SSR markers (named with 'SSR' as the prefix for distinction) and InDel markers based on their flanking sequences using the program Web-Premier (http://www.yeastgenome.org/cgi-bin/web-primer).

\subsection{Gene mapping}

The $\mathrm{F}_{2}$ population of esp $2 \times$ Xiushui-13 was used for gene mapping. Following the principle of bulked segregant analysis (BSA) [17], 15 normal plants and 15 mutant plants were randomly selected from the $F_{2}$ population to make 2 DNA pools, which were genotyped, with the 2 parents as controls, to quickly identify markers possibly linked to the target gene. The linked markers screened were further used to genotype individual mutant plants in the $F_{2}$ population, and the linkage relationship among the target gene and the markers was analyzed using the program Mapmaker/Exp 3.0 [18]. Total DNA extraction and molecular marker detection were all performed following the methods described by Duan et al. [19].

\subsection{Sequence analysis and candidate gene identification}

According to the annotations of the rice genome (http://rice. plantbiology.msu.edu/), all the genes located within the interval covering the target gene were found out, from which the candidate gene was identified. The candidate gene was further confirmed by sequencing analysis.

\section{Results}

\subsection{Morphological features of esp2}

The mutant esp2 had normal vegetative growth. Its phenotype was similar to that of the wild type (Minghui-86) at the seedling stage and the tillering stage. After heading stage, however, the mutant was obviously dwarfed, with panicles entirely enclosed by flag leaf sheaths and uppermost internodes hardly elongated (length $<0.3 \mathrm{~cm}$; whereas, length $\approx$ $30 \mathrm{~cm}$ in the wild type); nevertheless, the other internodes in the mutant had no significant changes in length (Figure 1). In addition, the panicle length of esp2 was also slightly 

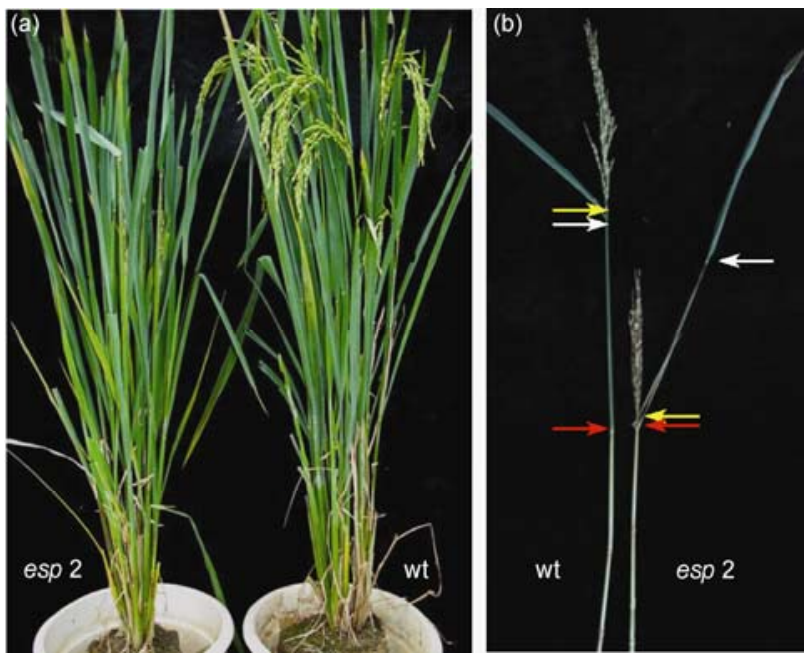

Figure 1 Phenotypes of the panicle enclosure mutant esp2 and the wild type in rice. (a) Plant; (b) panicle and upper part of stem. White arrow: flag leaf pulvinus; yellow arrow: panicle neck node; red arrow: uppermost node; esp2: mutant; wt: wild type.

( 14\%) shorter and the grain number per panicle of esp 2 was slightly $(12 \%)$ smaller than those of the wild type. Hence, we named the mutant as enclosed shorter panicle 2 (esp2; here, the number 2 was for distinction from another panicle enclosure mutant discovered by us). However, the fertility of esp 2 was not affected. The mutant could produce seeds normally.

\subsection{Inheritance of the mutant trait}

The $\mathrm{F}_{1}$ generations of the crosses between esp 2 mutant and Minghui-86, Zao-R974 and Xiushui-13 were all normal in heading. Their $F_{2}$ generations all showed the segregation of normal plants and mutant plants, following a ratio of $3: 1$ (Table 1), suggesting that the mutant phenotype is caused by a single recessive gene. Besides, the mutant characteristic of panicle enclosure was always typical in different genetic background, suggesting that the mutant phenotype can be inherited steadily.

\subsection{Preliminary mapping of ESP2}

A total of 165 RM-series SSR markers were used to analyze the two parents (esp2 and Xiushui-13) and the normal and mutant DNA pools. Three SSR markers on the end of the short arm of chromosome 1, RM84, RM1282 and RM495, were found to be polymorphic between the 2 pools, suggesting that they might be linked to the target gene ESP2. Further analysis with the three SSR markers on 770 mutants from the $\mathrm{F}_{2}$ population showed that ESP2 is located between RM1282 and RM84, with genetic distances of 1.01 and $10.29 \mathrm{cM}$ to the two markers, respectively (Figure 2).
Table 1 Segregations in the $\mathrm{F}_{2}$ populations of crosses between esp $2 \mathrm{mu}-$ tant and different cultivars

\begin{tabular}{ccccc}
\hline Cross combination & Normal plant & Mutant plant & $\chi^{2}(3: 1)$ & $P$ \\
\hline esp2/Minghui-86 & 302 & 105 & 0.14 & 0.70 \\
esp2/Zao-R974 & 427 & 147 & 0.11 & 0.70 \\
esp2/Xiushui-13 & 4225 & 1380 & 0.43 & 0.50 \\
\hline
\end{tabular}

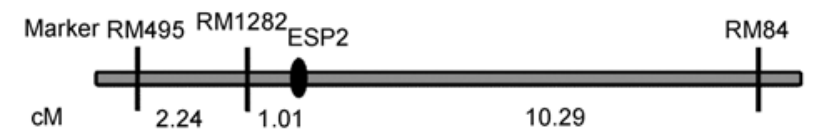

Figure 2 Result of preliminary mapping of ESP2.

\subsection{Fine mapping of ESP2}

Nine SSR markers were designed in the interval between RM1282 and RM84. Three of them (named SSR1, SSR2 and SSR3; see Table 2 for their primer sequences) showed polymorphisms between the 2 parents. Analysis of $770 \mathrm{~F}_{2}$ mutant plants with these three SSR markers indicated that ESP2 is located between SSR1 and SSR3 with distances of 0.50 and $2.67 \mathrm{cM}$ to the two markers, respectively, and cosegregated with SSR2. The BAC clone containing SSR2 (AP002541) and its two adjacent BAC clones (AP002747 and AP002868) were found out from the rice genome sequence, and 17 InDel markers were designed on these BAC clones by comparing the sequence differences between japonica rice (Nipponbare) and indica rice (93-11). Five of these InDel markers (named InDel1-InDel5; see Table 2 for their primer sequences) showed polymorphisms between the 2 parents. These five InDel markers together with SSR2 were employed to analyze $2720 \mathrm{~F}_{2}$ mutant plants. The results indicated that ESP2 was located between InDel2 and SSR2, with a genetic distance of $0.018 \mathrm{cM}$ (one recombinant), respectively, or a corresponding physical distance of $\sim 14 \mathrm{~kb}$ to either of the markers (Figure 3 ).

\subsection{Candidate of ESP2}

According to the rice genome sequence annotation provided by the rice genome database (http://rice.plantbiology.msu. edu/), there are only two genes in the $14-\mathrm{kb}$ region where ESP2 was located (Figure 3). One is a putative phosphatidylserine synthase gene, the other is a putative glycosyltransferase gene. As part of the sequence of the latter gene is located outside the 14-kb region, we thought that the former one might be the candidate gene of ESP2. This was supported by the results of sequencing analysis, which indicated that the putative phosphatidylserine synthase gene in the mutant was inserted by a 5287-bp retrotransposon sequence at $1296 \mathrm{bp}$ downstream from the initiation codon, while the putative glycosyltransferase gene sequence in the mutant was exactly the same as that in the wild type. 
Table 2 Markers and their primer sequences used for the fine mapping of ESP2

\begin{tabular}{clll}
\hline Marker & \multicolumn{1}{c}{ Forward $\left(5^{\prime} \rightarrow 3^{\prime}\right)$} & \multicolumn{1}{c}{ Reverse $\left(5^{\prime} \rightarrow 3^{\prime}\right)$} & BAC clone \\
\hline SSR1 & TTGCAATCCAAACCTCTGTG & AATCCTGCCTTCCTCCAGTT & AP003338 \\
Indel1 & CAGCAAGAGGGTTCAAGTAG & TTTAATGTGGGAAAGACACC & AP002541 \\
Indel2 & CACTCATGCCCCTTTGAAAT & TGGATTGACCGTAGTGGAGA & AP002541 \\
SSR2 & GGCTTCAGCTACCTTCTTCT & AGCAGGGAACTGAAAGAGAG & AP002541 \\
Indel3 & TGCAGCTCTGTACAAGGTAGT & TGCTTGATCTTCTTCAGGAC & AP002541 \\
Indel4 & GTTGTCATGTTCCTGTTTGC & CCAACTTTGACCTGTGATGT & AP002541 \\
Indel5 & AGGTGGAGCTATGCTTTGA & GCCTCCATGACATTATTTGT & AP002868 \\
SSR3 & TTGTGTGTGAATTGTGTTGG & GCAATCTGAGAGCGTTTTA & AP003214 \\
\hline
\end{tabular}

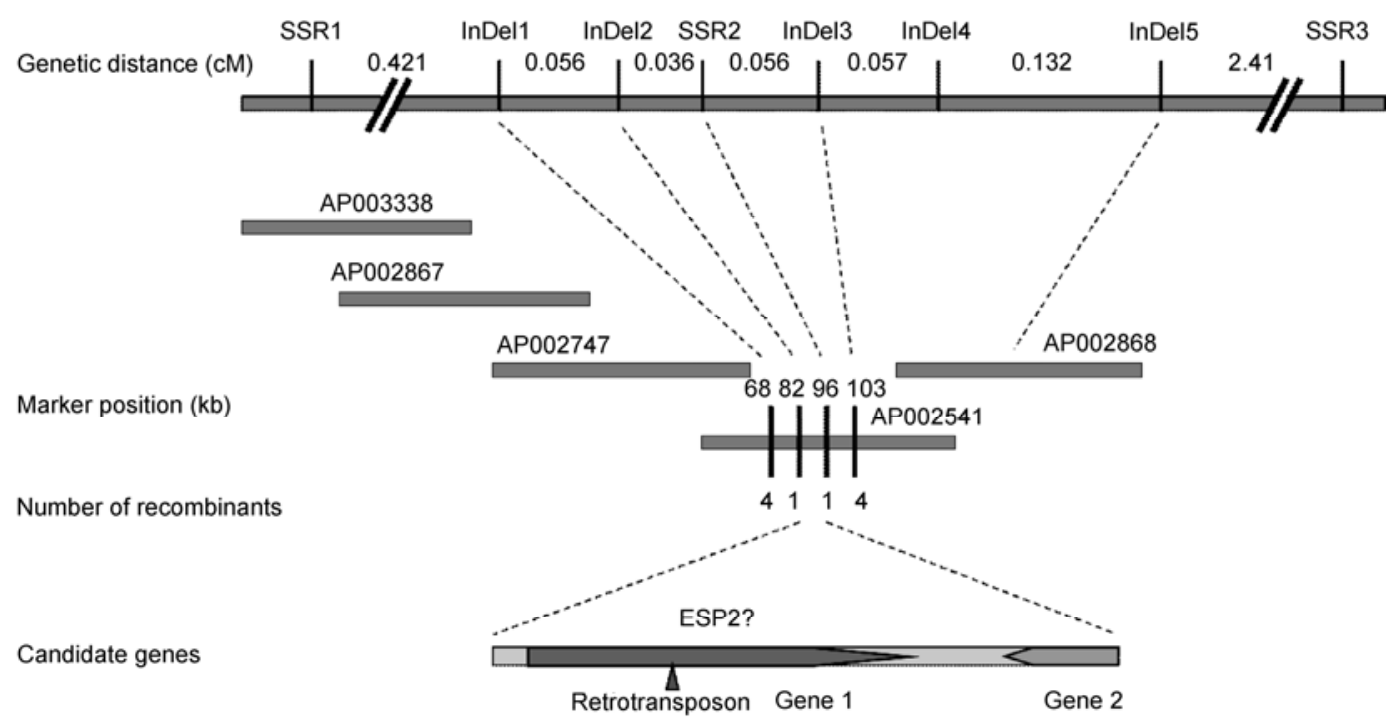

Figure 3 Fine mapping result and candidate gene identification of ESP2.

\section{Discussion}

The phenomenon of panicle enclosure in rice is mainly caused by the shortening of uppermost internode. The degree of panicle enclosure is determined by the degree of uppermost internode shortening. The mutant esp2 discovered in this study has two important features: (i) the uppermost internode is almost completely degenerated and hardly elongated, resulting in complete enclosure of the panicle inside the flag leaf sheath; and (ii) the internode shortening is highly specific, limited to the uppermost internode only, while all other internodes do not show obvious changes in length. These two features reflect the importance and specificity of ESP2 for the development of uppermost internode. According to the second feature, it appears that there is a regulation mechanism for the development and elongate of uppermost internode independent of other internodes. This mechanism might be related to the special position of uppermost internode (as a junction between panicle and stem) and its important role in the process of reproduction (heading). Therefore, deeply studying the functions of ESP2 is significant for understanding the molecular mechanisms of uppermost internode development and heading in rice.
At present, 5 enclosed panicle mutants have been reported in rice, including $s h p l, \operatorname{shp} 2, \operatorname{shp5}$, shp6 and $f_{s p}$ [13-16], most of which are expressed as partial panicle enclosure, completely different from esp2. Although $f_{s p}$ exhibits complete panicle enclosure, its uppermost five internodes are all shortened to some extent, unlike esp2 which only affects the uppermost internode specifically. So far, we have only seen a report that SHP6 gene was mapped on chromosome 2 [13], but not seen reports about the mapping of other panicle enclosure genes. Obviously, SHP6 is not allelic to ESP2. Taken together, esp2 should be a new enclosed panicle mutant and ESP2 should be the first panicle enclosure gene that has been fine mapped.

In this study, we fine mapped ESP2 to a $14-\mathrm{kb}$ region using a large population. According to the sequence annotation, only one intact gene exists in this region, namely, a putative phosphatidylserine synthase gene. Sequencing analysis on the mutant and the wild type suggested that the putative phosphatidylserine synthase gene is very likely to be ESP2. Phosphatidylserine is an important component of cell membrane phospholipids, which can regulate the functional status of key proteins in cell membrane, has important regulating effect on cellular metabolism, and acts as an in- 
termediate between cell membrane receptors and second receptors [20]. Phosphatidylserine synthase is a tool enzyme for synthesizing phosphatidylserine, belonging to the family of phosphatidyl diester synthase, which takes phospholipids as substrates, catalyzing transesterification between phospholipids and nucleophilic donors in the water [21]. Presently, functional phosphatidylserine synthase genes have been found in bacteria, yeast, mammals and plants. As a phosphatidylserine synthase gene, why ESP2 plays such an important and specific role in the development of uppermost internode in rice? This is really an interesting question. We are now studying the functions of the candidate of ESP2 in-depth from a number of aspects, hoping to find out the answer.

We thank Lin Lihui, Lan Tao, Zhao Jizhi, Zhang Xuzhang, Yang Fan, Guan Zhiqin, Tang Weiqi, Zhang Shiyuan, Chen Liping, Lin Yanqiu and Huang Ding of the College of Crop Science, Fujian Agriculture and Forestry University, for their assistance in the population construction. This work was supported by the National Transgenic Projects of China (2009ZX08009-109B), Natural Science Foundation of Fujian Province (2010J01077), the National High Technology Research and Development Program of China (2006AA10Z128) and New Century Excellent Talents in University of Fujian Province (KY0010057).

1 Wu C, Deng X J, Liu A Q, et al. Strategy of gene engineering in hybrid rice breeding in China (in Chinese). Biotechnol Bull, 2003, (1): 4-7

2 Deng H F, He Q, Shu F, et al. Status and technical strategy on development of japonica hybrid rice in China (in Chinese). Hybrid Rice, 2006, 21: 1-6

3 Li A X, Li Z H, Ding K X, et al. Measures for preventing and controlling kernel Smut in hybrid rice seed production (in Chinese). Jiangsu Agric Sci, 1995, 4: 34-36

4 Rutger J N, Camahan H L. A fourth genetic element to facilitate hybrid cereal productions recessive tall in rice. Crop Sci, 1981, 21: 373-376

5 Yang R C, Zhang S B, Huang R H, et al. Breeding technology of eui-hybrids of rice. Agr Sci Sin, 2002, 35: 233-237

$6 \mathrm{He} \mathrm{Z} \mathrm{H,} \mathrm{Li} \mathrm{D} \mathrm{B.} \mathrm{Relations} \mathrm{of} \mathrm{plant} \mathrm{height} \mathrm{genes} \mathrm{to} \mathrm{the} \mathrm{sensitivity} \mathrm{of}$ $\mathrm{GA}_{3}$ and to the regulation of endogenous in different rice growth stages. Plant Physiol Commun, 1994, 30: 170-174
7 Zhu H B. Fine mapping and cloning of rice EUI2 $(t)$ gene controlling upper most internode elongation. Doctor Dissertation. Fu Zhou: Fujian Agriculture and Forestry University, 2003

8 Zhu Y Y, Nomura T, Xu Y H, et al. Elongated uppermost internode encodes a cytochrome P450 monooxygenase that epoxidizes gibberellins in a novel deactivation reaction in rice. Plant Cell, 2006, 18: $442-456$

9 He Z H, Shen Z T. Sensitivity of elongated internode gene to $\mathrm{GA}_{3}$ and improvement of $\mathrm{ms}$ line in rice (in Chinese). Acta Agron Sin, 1994, 20: 161-167

10 Liang K J, Wang N Y, Yang R C. Inheritance and breeding utilization of panicle exsertion of rice (in Chinese). J Fujian Agric Univ (Nat Sci), 1992, 21: 380-385

11 Shen Z T, Yang C D, He Z H. Studies on eliminating panicle enclosure in WAType MS line of rice (Oryza sativa subsp. indica)(in Chinese). J Chi Rice Sic, 1987, 1: 95-99

12 Virmani S S, Dalmacio R D, Lopez M T. EUI gene for elongated uppermost internode transferred to indica rice. Int Rice Res Newsl, 1988, 13: 6-9

13 Zhu K M. Genetic analysis and mapping of SHP6 gene in rice (in Chinese). Master Dissertation. Yangzhou: Yangzhou University, 2006

14 Kinoshita T. Report of the committee on gene symbolization nomenclature and linkage groups. Rice Genet News, 1990, 7: 16-50

15 Liu Z, Luo L J. Anatomical studies on the stem of rice of dwarf and sheathed panicle (in Chinese). Chin Agri Sci Bull, 2006, 22: 409-412

16 Wang W P, Zhu F Z, Tang L, et al. Discovery and preliminary analysis of a rice mutant with fully sheathed panicle (in Chinese). Chin Agri Sci Bull, 2008, 24: 212-216

17 Michelmore R W, Paran I, Kesseli R V. Identification of markers linked to disease-resistance genes by bulked segregant analysis: A rapid method to detect markers in specific genomic regions by using segregating populations. Proc Natl Acad Sci USA, 1991, 88: 98289832

18 Lander E S, Green P, Abrahamson J, et al. MAPMAKER: An interactive computer package for constructing primary genetic linkage maps of experimental and natural populations. Genomics, 1987, 1: 174-181

19 Duan Y L, Wu W R, Liu H Q, et al. Genetic analysis and gene mapping of leafy head (lhd), a mutant blocking the differentiation of rachis branches in rice (Oryza sativa L.) (in Chinese). Chinese Sci Bull, 2003, 48: 2201-2205

20 Kidd P. Phosphatidylserine: Membrane nutrient for memory. A clinical and mechanistic assessment. Altern Med Rev, 1996, 1: 70-84

21 Leiros I, McSweeney S, Hough E. The reaction mechanism of phospholipase D from Streptomyces sp. Strain PMF. Snapshots along the reaction pathway reveal a pentacoordinate reaction intermediate and an unexpected final product. Mol Biol, 2004, 339: 805-820

Open Access This article is distributed under the terms of the Creative Commons Attribution License which permits any use, distribution, and reproduction in any medium, provided the original author(s) and source are credited. 